\title{
UNIPOTENT AND PROUNIPOTENT GROUPS: COHOMOLOGY AND PRESENTATIONS
}

\author{
BY ALEXANDER LUBOTZKY AND ANDY R. MAGID
}

A pro-affine algebraic group $G$, over the field $k$ (which we always take to be algebraically closed of characteristic zero) is an inverse limit of affine algebraic groups [3]. If the algebraic groups in the inverse system are unipotent, we call $G$ prounipotent. Pro-affine algebraic groups arise naturally in the theory of finite-dimensional $k$-representations of discrete and analytic groups $[3,4,9]$ and prounipotent groups arise naturally as the prounipotent radicals of pro-affine groups. Our interest in prounipotents is motivated by possible applications to finitedimensional representation theory.

The extension of the category of unipotent groups to that of prounipotents makes possible "combinatorial group theory" (free groups and presentations):

If $X$ is a set, there is a prounipotent group $F(X)$ containing $X$ such that for every prounipotent group $H$ and function $f: X \rightarrow H$ with $\operatorname{Card}\left\{X-f^{-1}(L)\right\}$ finite for every closed subgroup $L$ of finite codimension in $H$ there is a unique homomorphism $\bar{f}: F(X) \rightarrow H$ extending $f[5,2.1]$. Every prounipotent group $G$ is a homomorphic image of a free prounipotent group $F$ so there is an exact sequence (*) $1 \rightarrow R \rightarrow F \rightarrow G \rightarrow 1$. We can choose (*) with $R \subseteq(F, F)$ and in this case we call (*) a proper presentation of $G$. If $F=F(X)$ in (*), we call $X$ generators for $G$ and we call generators of $R$, as a prounipotent normal subgroup of $F$, relations for $G$.

As for pro- $p$ groups [11], the numbers of generators and relations for $G$ have a cohomological interpretation. Cohomology here is in the category of polynomial representations as in [2]. There is a unique simple in this category (the one-dimensional trivial module $k$ ) so cohomological dimension is defined as $\operatorname{cd}(G)$ $=\inf \left\{i \mid H^{n}(G, k)=0, n>i\right\}$.

THEOREM 1 [5, 2.8 AND 2.9]. The following are equivalent for prounipotent $G$ :

(a) $G$ is free,

(b) $G$ is a projective group in the category of prounipotent groups,

(c) $\operatorname{cd}(G) \leqslant 1$.

Received by the editors November 13, 1981.

1980 Mathematics Subject Classification. Primary 14L25, 20 G10. 
Proposition $2[5,1.14]$. If $H$ is a prounipotent subgroup of the prounipotent group $G$ then $\operatorname{cd}(H) \leqslant \operatorname{cd}(G)$.

Corollary $3[5,2.10]$. A closed subgroup of a free prounipotent group is free.

THEOREM 4 [5, 3.2 AND 3.11]. Let $1 \rightarrow R \rightarrow F(X) \rightarrow G \rightarrow 1$ be a proper presentation of the prounipotent group $G$. Then $d=\operatorname{dim}\left(H^{1}(G, k)\right)=$ $\operatorname{Card}(X)$ and $r=\operatorname{dim}\left(H^{2}(G, k)\right)$ is the minimal number of normal generators of $R$ as a prounipotent subgroup of $F$. Thus, $d$ is the minimal number of generators and $r$ is the minimal number of relations for $G$.

The preceding results are proved similarly to the analogous results for pro- $p$ groups. (See [11].) Special properties of prounipotents extablish

TheOREM 5 [5, 3.14]. If $G$ is prounipotent and $\operatorname{dim}\left(H^{n}(G, k)\right)=1$ for some $n \geqslant 1$, then $\operatorname{cd}(G)=n$.

If $G$ is one-relator, $\operatorname{dim}\left(H^{2}(G, k)\right)=1$ by Theorem 4 so

CoRollary $6[5,3.15]$. A one-relator prounipotent group has cohomological dimension 2.

(Corollary 6 is the prounipotent analogue of $[9,11.2$, p. 633].)

When $G$ is finite-dimensional, $\operatorname{cd}(G)=\operatorname{dim}(G)$, so the only one-relator $G$ is $k \times k$. In general, there is a Golod-Shafarevich type inequality relating the numbers of generators and relations.

THEOREM $7[7,3.11]$. Let $G, d$, and $r$ be as in Theorem 4 with $r \neq 0$ and $G$ finite-dimensional. Then $r \geqslant d^{2} / 4$, with strict inequality unless $G=$ $k \times k$, when $r=1$ and $d=2$.

The proof of Theorem 7 relies on the notion of a group algebra developed in [6 and 7]: The coordinate ring $k[G]$ of the prounipotent group $G$ is a $G$ bimodule so that the right translations define an embedding $\rho$ of $G$ in the units of the $G$-module endomorphism ring of $k[G]$ as a left $G$-module. We denote $\operatorname{End}_{G}(k[G])$ by $k\langle\langle G\rangle$.

When $G$ is finitely generated, $k\langle\langle G\rangle\rangle$ is like a group algebra for $G$ (if $B$ is a finite-dimensional associative algebra, $U_{1}(B)$ is the group of units of $B$ congruent to 1 modulo the radical).

THEOREM $8[7,2.8]$. If $G$ is a finitely generated prounipotent group and $B$ a finite-dimensional associative $k$-algebra any polynomial representation $G \rightarrow$ $U_{1}(B)$ extends uniquely to an algebra homomorphism $k\langle\langle G\rangle \rightarrow B$. Moreover, this property characterizes $k\langle\langle G\rangle\rangle$. 
THEOREM $9[7,2.10]$. Let $G$ be a prounipotent group with a proper presentation $1 \rightarrow R \rightarrow F\left(\left\{x_{1}, \ldots, x_{d}\right\}\right) \rightarrow G \rightarrow 1$ where $\left\{s_{1}, \ldots, s_{r}\right\}$ is a minimal set of normal generators of $R$. Then $k\langle\langle G\rangle$ is the formal (noncommutative) power series algebra $k\left\langle\left\langle\rho\left(x_{1}\right)-1, \ldots, \rho\left(x_{d}\right)-1\right\rangle\right.$ modulo the ideal generated by $\left\{\rho\left(s_{i}\right)-1\right\}$.

Theorem 9 is proved by first treating the case where $G$ is free on $\left\{x_{1}, \ldots\right.$, $\left.x_{d}\right\}[6,1.5]$ (so $k\langle\langle G\rangle$ is a formal power series algebra). Then the embedding $\rho: G \rightarrow k\langle\langle G\rangle\rangle$ embeds $G$ in the ring of formal power series. This extends (in fact, reproves) the Magnus embedding [1, p. 151] of the free discrete group, and provides a concrete description of the free prounipotent group on $d$ generators as the Zariski closure of the subgroup generated by $\left\{1+t_{i}\right\}$ in the group of units of constant term 1 in $\left.k\left\langle t_{1}, \ldots, t_{d}\right\rangle\right\rangle$. Using this description, we obtain

THEOREM $10[6,2.7]$. The associated graded Lie algebra [1, p. 145] of the lower central series of a free prounipotent group on $d$ generators is a free $k$ Lie algebra on $d$ generators.

The proofs of the preceding theorems use a description of $k[G]$ as an ascending union of $G$-submodules $E_{i}(G)$ defined by $E_{-1}(G)=0$ and $E_{i+1}(G) / E_{i}(G)$ $=\left(k[G] / E_{i}(G)\right)^{G}$. If $G$ is finitely generated then the numbers $c_{i}(G)=\operatorname{dim}\left(E_{i}(G)\right)$ are all finite, and we have

Proposition 11 [6, 1.3 AND 7, 3.12]. Let $G$ be prounipotent.

(a) $G$ is free on $d$ generators if and only if $c_{i}(G)=1+d+d^{2}+\cdots+$ $d^{i}$ for $i \geqslant 0$.

(b) $G$ is finite-dimensional if and only if the series $\left\{c_{i}(G)\right\}$ has polynomial growth.

Finally, we record some applications to the finite-dimensional representation theory of a discrete group $\Gamma$. We let $A(\Gamma)$ be the pro-algebraic hull of $\Gamma[10,2.2]$ and $R_{u}(\Gamma)$ the prounipotent radical of $A(\Gamma)$.

THEOREM $12[5,4.3]$. If $\Gamma$ contains a free subgroup of finite index, $R_{u}(\Gamma)$ is a free unipotent group.

If $\Gamma$ is torsion free nilpotent, then $R_{u}(\Gamma)$ is finite-dimensional, and there is an embedding $\Gamma \rightarrow R_{u}(\Gamma)$. (This is the Malcev embedding for which our methods provide a new proof $[6,5.12]$.) In this case we have $H^{i}(\Gamma, k)=H^{i}\left(R_{u}(\Gamma), k\right)$ $[7,3.8]$ so we can apply Theorem 7 to obtain an inequality relating the ranks of the first and second cohomology groups of $\Gamma$.

\section{REFERENCES}

1. N. Bourbaki, Lie groups and Lie algebras. I, Addison-Wesley, Reading, Mass., 1975. $492-579$.

2. G. Hochschild, Cohomology of algebraic linear groups, Illinois J. Math. 5 (1961), 
3. G. Hochschild and G. D. Mostow, Pro-affine algebraic groups, Amer. J. Math. 91 (1964), 1127-1140. 663-689.

4. A. Lubotzky, Tannaka duality for discrete groups, Amer. J. Math. 102 (1980),

5. A. Lubotzky and A. Magid, Cohomology of unipotent and prounipotent groups, J. Algebra: 74 (1982), 76-95.

6. - Free prounipotent groups (preprint).

7. The group algebra of a prounipotent group (preprint).

8. R. Lyndon, Cohomology theory of groups with a single defining relation, Ann. of Math. (2) 52 (1960), 650-665.

9. A. Magid, Module categories of analytic groups, Cambridge Univ. Press, London and New York (to appear).

10. G. D. Mostow, Representative functions on discrete groups and solvable arithmetic groups, Amer. J. Math. 92 (1970), 1-32.

11. J. P. Serre, Cohomologie galoisienne, Lecture Notes in Math., vol. 5, SpringerVerlag, Berlin and New York, 1965. ISRAEL

DEPARTMENT OF MATHEMATICS, BAR-ILAN UNIVERSITY, RAMAT GAN,

DEPARTMENT OF MATHEMATICS, UNIVERSITY OF OKLAHOMA, NORMAN, OKLAHOMA 73019 